\title{
MITOCHONDRIAL TRNA VALINE AS A RECURRENT TARGET FOR MUTATIONS INVOLVED IN MITOCHONDRIAL CARDIOMYOPATHIES
}

Juan J. Arredondo ${ }^{\mathrm{a}^{*}}$, M. Esther Gallardo ${ }^{\mathrm{a}, \mathrm{b}^{*}}$, Pablo García-Pavía ${ }^{\mathrm{c}, \mathrm{d}}$, Verónica Domingo ${ }^{\mathrm{a}}$, Begoña Bretón ${ }^{\mathrm{a}}$, M. Teresa García-Silva ${ }^{\mathrm{e}}$, M. Jesús Sedano ${ }^{\mathrm{f}}$, Miguel A. Martín ${ }^{\mathrm{g}, \mathrm{b}}$, Joaquín Arenas ${ }^{\mathrm{g}, \mathrm{b}}$, Margarita Cervera ${ }^{\mathrm{a}}$, Rafael Garesse ${ }^{\mathrm{a}, \mathrm{b}}$, Belén Bornstein ${ }^{\mathrm{a}, \mathrm{b}, \mathrm{h}, \#}$

${ }^{a}$ Departamento de Bioquímica, Instituto de Investigaciones Biomédicas Alberto Sols CSIC-UAM, Facultad de Medicina, Universidad Autónoma de Madrid, Spain

b Centro de Investigación Biomédica en Red de enfermedades raras (CIBERER), Madrid, Spain

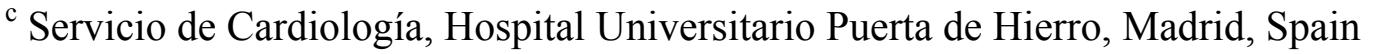

d Red de Investigación Clínica y Básica en Insuficiencia Cardíaca (REDINSCOR), Madrid, Spain

e Servicio de Pediatría, Hospital Universitario 12 de Octubre, Madrid, Spain

${ }^{\mathrm{f}}$ Servicio de Neurología, Hospital General Yagüe, Burgos, Spain

g Laboratorio de enfermedades mitocondriales, Centro de Investigación, Hospital Universitario 12 de Octubre, Madrid, Spain

${ }^{\mathrm{h}}$ Servicio de Bioquímica, Hospital Universitario Puerta de Hierro, Madrid, Spain

* These two authors contributed equally to this work

\# Corresponding author: Departamento de Bioquímica, Instituto de Investigaciones Biomédicas "Alberto Sols" CSIC-UAM, Universidad Autónoma de Madrid, c/Arzobispo Morcillo 4, 28029 Madrid, Spain. Phone: +34-91-4975452; Fax: +34-915854401; email: belen.bornstein@uam.es 


\begin{abstract}
The aim of this study was to identify the genetic defect in two patients having cardiac dysfunction accompanied by neurological symptoms, and in one case MRI evidence of cortical and cerebellar atrophy with hyperintensities in the basal ganglia. Muscle biopsies from each patient revealed single and combined mitochondrial respiratory chain deficiency. The complete mtDNA sequencing of both patients revealed two transitions in the mitochondrial tRNA ${ }^{\mathrm{Val}}$ gene $(M T-T V)(\mathrm{m} .1628 \mathrm{C}>\mathrm{T}$ in patient 1 , and $\mathrm{m} .1644 \mathrm{G}>\mathrm{A}$ in patient 2 ). The functional and molecular analyses reported here suggest that the $M T-T V$ gene should be routinely considered in the diagnosis of mitochondrial cardiomyopathies.
\end{abstract}

Keywords: Mitochondrial DNA, m.1628C $>\mathrm{T}, \mathrm{m} .1644 \mathrm{G}>\mathrm{A}$, mitochondrial tRNA quantification, mitochondrial diseases, mitochondrial cardiomyopathies

\title{
Abbreviations:
}

MD, mitochondrial disease, mtDNA, mitochondrial DNA; tRNA, transfer ribonucleic acid, MCM, mitochondrial cardiomyopathy, MELAS, mitochondrial encephalopathy, lactic acidosis and stroke-like episodes; MiMyCa, maternally inherited myopathy and cardiomyopathy; MNGIE, mitochondrial neurogastrointestinal encephalomyopathy; mttRNA, mitochondrial tRNA; CS, citrate synthase; bp, base pairs; RFLP, restriction fragment length polymorphism; nt, nucleotide; MRC, mitochondrial respiratory chain; ECG, electrocardiogram. 


\section{Introduction}

Mitochondrial diseases (MDs) are a diverse group of disorders characterized by defects of the mitochondrial respiratory chain (DiMauro et al., 2008; Tucker et al., 2010). These MDs can present at any age, and their clinical symptoms reflect a primary defect in tissues with high oxidative demand. The predominance of neuromuscular manifestations in MDs usually masks the presence of other clinical phenotypes, such as cardiac complications, but mitochondrial defects are being increasingly recognized to play a role in the pathogenesis of hereditary cardiomyopathies (Led et al., 2004). Sporadic or inherited mutations in mitochondrial DNA (mtDNA), specifically in the mtDNA transfer ribonucleic acid (tRNA) genes, or in the nuclear genome have been associated with hypertrophic and dilated cardiomyopathy (Papadopoulou et al., 1999; Hirano et al., 2001; Bénit et al., 2003; Taylor et al., 2003). Cardiac conduction abnormalities have also been associated with different mtDNA rearrangements (MarínGarcía et al., 2002). Hence, the cardiac and neuromuscular manifestations of these mutations share common pathophysiological processes, and mitochondrial cardiomyopathies (MCMs) can be either heart-specific or associated with a spectrum of other symptoms (Led et al., 2004). However, heart-related manifestations in these patients are relatively mild compared to the severity of their neurological symptoms and the generalized muscle weakness that limits their activity.

One of the first mtDNA point mutations associated with a maternally transmitted MCM and myopathy was described in 1991 (Zeviani et al., 1991), and the acronym MiMyCa was coined for this clinical syndrome. Since then, several mutations in the mitochondrial genome, mainly point mutations in mitochondrial tRNA (mt-tRNA) genes, have been associated with different MCM phenotypes (Hirano et al., 2001; Taylor et al., 2003.). In an attempt to identify mtDNA mutations associated with 
MCMs, we performed a systematic search in a series of patients that were suspected of MCM. As a result of this study, two transitions in the $M T-T V$ gene were identified, the putative m.1628C $>\mathrm{T}$ mutation and the previously described, but not functionally characterized, m.1644G $>$ A sequence variation (Tanji et al., 2008). In the present report, a selective reduction of the steady-state mt-tRNA ${ }^{\mathrm{Val}}$ level in the patient harbouring the m.1644G $>$ A transition was found, confirming both the cardiac and the neurological involvement of this mutation.

\section{Material and methods}

\subsection{Patient histories}

\subsubsection{Patient 1}

This 14-year-old boy was the sixth child of healthy and non-consanguineous Moroccan parents who had 7 children, all healthy except for one sister who died for of an unknown cause and another younger sister who presented short stature. Our patient presented short stature during the first years of life. At 9 years of age the parents observed palpebral ptosis, which required surgery. Physical examination revealed external ophthalmoplegia, pigmentary retinitis, and significant muscle atrophy in legs and arms. Cardiology study showed sinus rhythm and incomplete right bundle branch block in the electrocardiogram and mild hypertrophic cardiomyopathy in a transthoracic echocardiogram. Electromyogram showed a myopathic pattern. Serum laboratory parameters were normal. Likewise, urinary organic acids, plasma amino acids, and acylcarnitine profile were normal. Muscle biopsy was performed based on suspicion of a mitochondrial disorder. 


\subsubsection{Patient 2}

This 32-year-old man reported that since the age of 21 he had progressive gastrointestinal dysmotility, manifesting as episodic abdominal pain with diarrhea and progressive weight loss. At the same time, he developed progressive dementia, loss of balance, and a diagnosis of progressive encephalopathy was established. The patient was cachectic (weight: 42 kilograms, height: $175 \mathrm{~cm}$ ) and physical examination showed mild difficulty to walk, with tandem gait. Examination of cognitive functions, which were clearly impaired, was made difficult by behavioural abnormalities. He had generalized muscle weakness and sensory neuropathy in the extremities with absent tendon reflexes. Ten years later, he was referred to our center because of a new intestinal obstruction crisis, fever, refractory seizures, and loss of consciousness. He was admitted to the intensive care unit and a viral meningoencephalitis was suspected. The patient was infected with a respiratory Pseudomonas aeruginosa and the neurological situation became critical. His clinical condition progressed rapidly and he died of respiratory insufficiency 5 days later.

Laboratory findings included normal serum creatine kinase, and high serum lactate $(9.7 \mathrm{mM}$; control values $<2 \mathrm{mM})$. The electrocardiogram revealed sinus rhythm and left bundle branch block, and the echocardiogram showed pronounced cardiomegaly and biventricular hypertrophy. Brain magnetic resonance imaging revealed mild diffuse cortical atrophy and severe cerebellar atrophy, and hyperintensities in the basal ganglia were found on $\mathrm{T}_{2}$ sequences. Muscle biopsy was performed based on suspicion of a mitochondrial disorder. 


\subsection{Muscle histochemistry and enzyme activities of mitochondrial respiratory chain}

complexes

Samples from patients and controls were obtained in accordance with the Helsinki Declaration of 1975 , as revised in 2000. Informed consent was obtained from the families. The Ethics Committees of the 12 de Octubre Hospital and the Puerta de Hierro Hospital approved the study.

Quadriceps muscle biopsies were performed under local anesthesia. Serial frozen muscle sections were histochemically investigated for the presence of MD as described previously (Sciacco et al., 1996). Gomori trichrome and succinate dehydrogenase stains were used to detect ragged red fibers, and they were scored as positive when seen in $>1 \%$ of all fibers. The cytochrome oxidase stain was used to detect complex IV activity. Respiratory chain complexes I-IV activities were measured in total muscle homogenates as described previously (Martinez et al., 2001), and related to those of citrate synthase (CS) to correct for mitochondrial volume.

\subsection{Mitochondrial DNA sequencing}

Total genomic DNA was extracted from skeletal muscle by standard protocols. The complete mtDNA was amplified from total DNA in 24 overlapping 800-1000-bplong PCR fragments. Primers were carefully designed using the revised human mitochondrial DNA Cambridge reference sequence (Anderson et al., 1981; Andrews et al., 1999). The PCR fragments were sequenced on both strands using an ABI 3710 (Applied Biosystems; Foster City, CA) sequencer and a dye terminator cycle sequencing kit (Applera, Rockville, MD). 
2.4 PCR/restriction fragment length polymorphism (RFLP) analysis

The level of the m.1628C $>\mathrm{T}$ and $\mathrm{m} .1644 \mathrm{G}>\mathrm{A}$ heteroplasmy in the $M T-T V$ gene were determined by the last-hot-cycle technique as described previously (Moraes et al., 1993). To screen for the novel m.1628C $>$ T transition, a 434-bp fragment was amplified by PCR using primers corresponding to nucleotide (nt) positions 1226-1245 (forward) and 1629-1660 (backward, primer mismatches in positions 1630 and 1633). PCR conditions were $95^{\circ} \mathrm{C}$ for $30 \mathrm{~s}, 61^{\circ} \mathrm{C}$ for $30 \mathrm{~s}$, and $72^{\circ} \mathrm{C}$ for $30 \mathrm{~s}$. After 35 cycles, $4 \mu \mathrm{Ci}$ of $\alpha^{32} \mathrm{P}-\mathrm{dCTP}(2500 \mathrm{Ci} / \mathrm{mmol})$ was added and a last cycle was performed. Aliquots $(10 \mu \mathrm{l})$ of PCR products were digested with $P v u I I$ overnight and electrophoresed on a $15 \%$ nondenaturing polyacrylamide gel. In wild type DNA, the enzyme cuts the 434 bp-PCR product into two fragments of 403-bp and 32-bp. The restriction site is absent when the $\mathrm{m} .1628 \mathrm{C}>\mathrm{T}$ transition is present. For the $\mathrm{m} .1644 \mathrm{G}>\mathrm{A}$ mutation, we used primers corresponding to nt positions 1585-1603 (forward) and 1757-1776 (backward) to PCRamplify a 192-bp fragment. In the presence of the mutation, this amplicon contains a restriction site for Tsp509I that produces two fragments of 133-bp and 59-bp. This restriction site is absent in the wild type molecule.

\subsection{Quantification of mitochondrial $t R N A^{\text {Val }}$}

mt-tRNA $^{\mathrm{Val}}$ was quantified as described previously (Bornstein et al., 2005). Briefly, total RNA $(5 \mu \mathrm{g})$ extracted from skeletal muscle was incubated at $90^{\circ} \mathrm{C}$ for 5 min, electrophoresed through a $12 \%$ polyacrylamide- $7 \mathrm{M}$ urea gel and electroblotted onto a Zeta-probe membrane. Hybridization was performed at $42^{\circ} \mathrm{C}$ for $24 \mathrm{~h}$. The tRNA $^{\mathrm{Val}}$ was detected using a synthetic 21-mer oligonucleotide (spanning mtDNA nucleotide positions 1669-1649), which had been labelled with $[\gamma-32 \mathrm{P}]$ ATP at its 5end. This oligonucleotide probe does not encompass the mutated sites. After 
hybridization, the blot was washed and the membrane was stripped and rehybridized under the same conditions with a $\left[\begin{array}{ll}\gamma & -32 \mathrm{P}]\end{array}\right.$ 5-end-labelled 21-mer oligonucleotide corresponding to mtDNA positions $597-577$ of the MT-TF gene. The ratio of tRNA ${ }^{\mathrm{Val}}$ to tRNA $^{\text {Phe }}$ was calculated by quantification of the respective signals by phosphorimager analysis.

\subsection{Cardiac evaluation}

Cardiac study included standard 12-lead ECG and transthoracic two-dimensional echocardiogram. Echocardiography was performed in the left lateral decubitus position using a Philips iE33 system (Philips Medical Systems, Eindhoven, The Netherlands). Standard techniques were used to obtain M-mode, two-dimensional, and Doppler measurements (Limongelli et al., 2010). The mean of three measurements was taken for each echocardiographic parameter. Patterns of hypertrophy were defined in accordance with previously published methods (Limongelli et al., 2010).

\section{Results}

\subsection{Histochemical and biochemical studies}

Muscle biopsy was performed based on suspicion of a mitochondrial disorder in both patients, and appeared normal both morphologically and histochemically. The activity of the different complexes of the mitochondrial respiratory chain (MRC) was assessed in muscle homogenates obtained from the biopsies. Patient 1 showed a single enzyme defect of complex I and an important increase in the activity of citrate synthase in his skeletal muscle. Patient 2 showed a combined enzyme defect of complexes I and IV of the MRC, and also a high citrate synthase activity in his skeletal muscle (Table 1). 


\subsection{Sequencing and PCR-RFLP analyses}

In order to identify putative mutations in the mitochondrial genome, we purified and completely sequenced the mtDNA obtained from skeletal muscle of both patients. Sequence analysis revealed a total of 49 nucleotide changes from the revised Anderson mtDNA sequence in patient 1 (Suppl Table 1A) (Anderson et al., 1981; Andrews et al., 1999). Haplogroup analysis showed the African origin of this subject, belonging to mitochondrial haplogroup L2a1. All sequence variations identified in this patient were previously described polymorphisms or synonymous substitutions within expressed sequences, except for the m.1628C $>\mathrm{T}$ transition identified in the $M T-T V$ gene. This mtvariant was shown to be homoplasmic, involving all copies of the mtDNA in skeletal muscle from patient 1 (Figure 1A).

On the other hand, complete sequencing of the mtDNA from patient 2 revealed 35 sequence variations from the revised Anderson mtDNA sequence (Suppl Table 1B), and indicated that this patient has a European origin (haplogroup X2b). Among the 35 mitochondrial variations, a previously described mutation, the $\mathrm{m} .1644 \mathrm{G}>\mathrm{A}$ mutation in the $M T-T V$ gene, was identified (Tanji et al., 2008). This sequence change was found in heteroplasmy, and the patient harboured $94.4 \%$ mutant mtDNA in his skeletal muscle (Figure 1B).

Since this is a retrospective study, we could not verify the presence of the two transitions in other tissues from each of the two patients, and therefore we could not determine the heteroplasmy percentages of the mutated $M T-T V$ gene in cardiac muscle, which may have given indications about the likely pathogenicity of these mt-variations.

\subsection{Mitochondrial tRNA ${ }^{\text {Val }}$ steady-state levels}

In order to study the potential pathogenicity of both mutations we decided to 
investigate the steady-sate level of tRNA ${ }^{\mathrm{Val}}$. Unfortunately, this study could only be carried out for the $\mathrm{m} .1644 \mathrm{G}>\mathrm{A}$ mutation, because of we ran out of muscle tissue of patient 1. Total RNA was extracted from a muscle sample of patient 2 and from two control samples, and resolved by polyacrylamide gel electrophoresis. After electroblotting, the membrane was hybridized to specific mt-tRNA ${ }^{\text {Val }}$ or control oligonucleotide probes as described in the Material and Methods section. Figure 2 shows the ratio of mt-tRNA ${ }^{\mathrm{Val}}$ to $\mathrm{mt}_{-\mathrm{RNA}}{ }^{\mathrm{Phe}}$ in patient 2 and control muscle samples. Quantitative analysis of the autoradiograms showed a significant $67 \%$ decrease in the steady-state level of the tRNA ${ }^{\mathrm{Val}}$ harbouring the m.1644G $>$ A mutation as compared to control samples (Figure 2).

\section{Discussion}

In this report, the clinical, histochemical, biochemical, and molecular diagnosis of two patients with suspected heart defect as a consequence of MD is described. This study revealed two different nucleotide variants in the $M T-T V$ gene: $\mathrm{m} .1628 \mathrm{C}>\mathrm{T}$ and $\mathrm{m} .1644 \mathrm{G}>\mathrm{A}$. A growing number of mitochondrial cardiomyopathies are found to be associated with point mutations in the mt-tRNA genes, especially $M T-T L 1, M T-T I$, and MT-TK (Marín-García et al., 2000; Led et al., 2004; Wahbi et al., 2010). MDs caused by mutations in the $M T-T V$ gene are not very frequent, but three of the seven point mutations described so far in this gene (Figure 3) have been associated with hypertrophic cardiomyopathy or severe cardiac failure (Chalmers et al., 1997; McFarland et al., 2002; Blakely et al., 2004). In accordance with these three reported cases, our two patients developed cardiac conduction abnormalities and hypertrophic cardiomyopathy within a neurological presentation, and because of this, together with radiographic findings, MD was suspected in both cases. Muscle biopsies did not 
confirm the clinical impression of $\mathrm{MD}$ as they were morphologically and histochemically normal in the two patients, in contrast with biopsy findings of patients with mutations in the $M T-T V$ gene previously described (Chalmers et al., 1997; Tiranti et al., 1998; Sacconi et al., 2002; McFarland et al., 2002; Tanji et al., 2008; Horváth et al., 2009; Yan et al., 2010). On the other hand, biochemical analysis showed a decrease in MRC complex I activity in muscle homogenate from patient 1, and a combined deficiency in the activity of MRC complexes I and IV from patient 2 , as in most previous reports on mutations in the $M T-T V$ gene (Chalmers et al., 1997; Tiranti et al., 1998; Sacconi et al., 2002; McFarland et al., 2002; Tanji et al., 2008; Horváth et al., 2009; Yan et al., 2010).

Complete sequencing of the mtDNA from muscle of patient 1 allowed us to identify a homoplasmic m.1628C $>\mathrm{T}$ transition in the $M T-T V$ gene. Although the presence of heteroplasmy is a common pathogenic criterion for mt-tRNA mutations (McFarland et al., 2004), homoplasmic mutations, as is the case of the m.1628C $>\mathrm{T}$ transition, are being increasingly recognized as causes of MD (McFarland et al., 2002; DiMauro, 2006; DiMauro, 2011). Although funcional analysis of this mutation was not possible due to the lack of sample, molecular data support that this nucleotide variation could be relevant from a functional point of view. Supporting data include: (i) the mutation is associated with a mitochondrial phenotype of hypertrophic cardiomyopathy with palpebral ptosis, external ophthalmoplegia, pigmentary retinitis, and muscle atrophy; (ii) a single enzyme deficiency of MRC complex I and an increase in the activity of citrate synthase in skeletal muscle; (iii) the mutation involves a $\mathrm{C}$ to $\mathrm{T}$ change in the anticodon-stem of the $M T-T V$ gene, which might interrupt the secondary structure of the anticodon-stem; (iv) mitochondrial nucleotide 1628 is quite conserved through evolution (Sprinzl et al., 1989; McFarland et al., 2004), suggesting that the m.1628C $>$ T 
sequence variation may have a functional consequence; (v) this mutation was identified only once in a total of more than 4000 mtDNA sequences from public databases (Ingman and Gyllensten, 2003) and in more than 600 mtDNA sequences from our laboratory, which include controls and samples from a wide spectrum of pathological conditions associated to mitochondrial dysfunctions; and vi) as an indication for suspecting its pathogenic character, the other sample in which the $\mathrm{m} .1628 \mathrm{C}>\mathrm{T}$ sequence variation was identified corresponds to a different haplogroup (haplogroup HV) (Torroni et al., 2003; Gallardo et al., 2006; DiMauro, 2011). In consequence, we propose that, in the absence of functional studies, the $\mathrm{m} .1628 \mathrm{C}>\mathrm{T}$ transition in the $M T$ $T V$ gene is a good candidate to be responsible for the clinical phenotype observed in patient 1 .

In patient 2 , the heteroplasmic $\mathrm{m} .1644 \mathrm{G}>\mathrm{A}$ transition was identified in skeletal muscle. Besides cardiac dysfunction, neurogastrointestinal manifestations were the main clinical phenotype in this patient. Gastrointestinal dismotility is not usually associated with mt-tRNA mutations, but it has been described in patients with MELAS caused by the mtDNA point mutation m.3243A $>\mathrm{G}$ (Shimotake et al., 1998; GarcíaVelasco et al., 2003). Recently, the m.1630A $>\mathrm{G}$ mutation in the $M T-T V$ gene was reported in a child with MNGIE-like disease (Horváth et al., 2009). On the other hand, the $\mathrm{m} .1644 \mathrm{G}>\mathrm{A}$ mutation was recently described in another patient, but with a different clinical phenotype (Tanji et al., 2008). Our patient presented a more severe clinical phenotype, including cardiac involvement, which was absent in the aforementioned case report; this could be due to a higher heteroplasmy percentage in the muscle of our patient $(94.4 \%)$ compared to that observed in the previously described patient $(85 \%)$ (Tanji et al., 2008). In addition, it is worth mentioning that the heteroplasmy percentage is higher in skeletal muscle of patients with MCMs and mutations in the MT-TV gene 
(Chalmers et al., 1997; McFarland et al., 2002; Blakely et al., 2004) than in patients without heart defects and mutations in the same gene (Taylor et al., 1996; De Coo et al., 1998; Tiranti et al., 1998; Sacconi et al., 2002; Tanji et al., 2008; Horváth et al., 2009). Hence, mutant levels in skeletal muscle above $94 \%$ are probably required to affect the cardiac muscle in patients with MD associated with mutations in the $M T-T V$ gene (Table 2).

To unravel the pathogenic mechanism of the m.1644G $>$ A mutation, the mitochondrial tRNA ${ }^{\mathrm{Val}}$ steady state levels were studied. As shown in Figure 2, there was a marked and selective reduction of the steady-state mt-tRNA ${ }^{\mathrm{Val}}$ level as compared to mt-tRNA ${ }^{\text {Phe }}$ in skeletal muscle from patient 2 (27\% of control values). Recent studies by us and others have shown that a decreased level of different mt-tRNAs inhibit mitochondrial protein synthesis (Enriquez et al., 1995; McFarland et al., 2002; Bornstein et al., 2005). Accordingly, patient 2 presents a strong reduction in complex I and IV activities in muscle homogenate. Moreover, our data are consistent with the m.1644G $>$ A mt-tRNA ${ }^{\text {Val }}$ being rapidly degraded. To date, the most pronounced decrease of the steady-state mt-tRNA ${ }^{\mathrm{Val}}$ in cardiac and in skeletal muscles has been observed in the presence of the mutation m.1624C $>\mathrm{T}$ (McFarland et al., 2002). This mutation, affecting the same mt-gene, is associated with infantile and fatal Leigh syndrome together with severe cardiac failure in one of ten children affected (McFarland et al., 2002). Interestingly, tissues from all members of this pedigree were homoplasmic for the $\mathrm{m} .1624 \mathrm{C}>\mathrm{T}$ mutation, as is the $\mathrm{m} .1628 \mathrm{C}>\mathrm{T}$ transition in the skeletal muscle of patient 1 in our study. However, the marked variability in phenotype among different family members can not be explained by this mtDNA defect alone (Rorbach et al., 2008). Our patient 2 had no known maternal family diagnosed of MD, and we could not confirm the presence of a low level mt-tRNA ${ }^{\mathrm{Val}}$ in cardiac muscle or 
in tissues from other maternal relatives. In addition, conformational changes or aminoacylation defects have also been found in other mt-tRNA point mutations as potential pathogenic mechanisms responsible for different clinical phenotypes (Enriquez et al., 1995; Bacman et al., 2003; Florentz et al., 2003; Bornstein et al., 2005). In our case, there was insufficient muscle sample and it could not be confirmed whether this mutation may also lead to a conformational change or impaired aminoacylation capacity of the mt-tRNA ${ }^{\mathrm{Val}}$.

In conclusion, the two cases presented in this report reinforce the notion that cardiac manifestations are frequent in patients with mitochondrial disorders. Therefore, cardiac evaluation should be routinely performed at the time of diagnosis of MD in these patients. This study also shows that mitochondrial tRNA mutations associated with heart disease not only involve the leucine, lysine, or isoleucine tRNA genes, and that the mitochondrial valine tRNA gene should be sequenced when there is a high suspicion of mitochondrial cardiomyopathy.

\section{Acknowledgements}

This work was supported by Grants PI060205 (to B.B.), PI070167 (to R.G.), and PI060547 (to M.A.M.) from the Instituto de Salud Carlos III (ISCIII), Ministry of Science and Innovation (MICIN); and GEN-0269/2006 from the Comunidad de Madrid (CM) (to M.A.M. and R.G.). M.E.G. is a Senior Fellow and receives grant support from the Center for Biomedical Research on Rares Diseases (CIBERER). B.B. is supported by the Program Intensificación de la Actividad Investigadora from the ISCIII-MICIN and the Agencia Lain Entralgo (CM). Members from CIBERER units U717 and U723 participated in this work. 
Conflict of Interest: none declared

\section{References}

Anderson, S., et al., 1981. Sequence and organization of the human mitochondrial genome. Nature 290, 457-465

Andrews, R.M., et al., 1999. Reanalysis and revision of the Cambridge reference sequence for human mitochondrial DNA. Nat. Genet. 23, 147

Bacman, S.R., Atencio, D.P., Moraes, C.T., 2003. Decreased mitochondrial tRNA ${ }^{\text {Lys }}$ steady-state levels and aminoacylation are associated with the pathogenic G8313A mitochondrial DNA mutation. Biochem. J. 374, 131-136

Bénit, P., et al., 2003. Mutant NDUFV2 subunit of mitochondrial complex I causes early onset hypertrophic cardiomyopathy and encephalopathy. Hum. Mutat. 21, $582-586$

Blakely, E.L., et al., 2004. Childhood neurological presentation of a novel mitochondrial tRNA $^{\text {Val }}$ gene mutation. J. Neurol. Sci. 225, 99-103

Bornstein, B., et al., 2005. Comparative analysis of the pathogenic mechanisms associated with the G8363A and A8296G mutations in the mitochondrial tRNA $^{\text {Lys }}$ gene. Biochem. J. 387, 773-778

Chalmers, R.M., et al., 1997. A mitochondrial DNA tRNA ${ }^{\mathrm{Val}}$ point mutation associated with adult-onset Leigh syndrome. Neurology 49, 589-592

De Coo, I.F.M., et al., 1998. A mitochondrial tRNA ${ }^{\mathrm{Val}}$ gene mutation (G1642A) in a patient with mitochondrial myopathy, lactic acidosis, and stroke-like episodes. Neurology 50, 293-295

DiMauro, S. 2006. Mitochondrial myopathies. Curr. Opin. Rheumatol. 18, 636-641

DiMauro, S., Schon, E.A., 2008. Mitochondrial disorders in the nervous system. Annu. 
Rev. Neurosci. 31, 91-123

DiMauro, S., 2011. Pathogenesis and treatment of mitochondrial myopathies: recent advances. Acta Myol. 29, 333-338

Enriquez, J.A., Chomyn, A., Attardi, G., 1995. MtDNA mutation in MERFF syndrome causes defective aminoacylation of Trna(Lys) and premature translation termination. Nat. Genet. 10, 47-55

Florentz, C., et al., 2003. Human mitochondrial tRNAs in health and disease. Cell. Mol. Life. Sci. $60,1356-1375$

Gallardo, M.E., et al., 2006. m.6267G $>$ A: a recurrent mutation in the human mitochondrial DNA that reduces cytochrome $\mathrm{C}$ oxidase activity and is associated with tumors. Hum. Mutat. 27, 575-582

García-Velasco, A., Gómez-Escalonilla, C., Guerra-Vales, J.M., Cabello, A., Campos, Y., Arenas, J. 2003. Intestinal pseudo-obstruction and urinary retention: cardinal features of a mitochondrial DNA-related disease. J. Intern. Med. 253, 381-385

Horváth, R., et al. 2009. Heteroplasmic mutation in the anticodon-stem of mitchondrial tRNA $^{\text {Val }}$ causing MNGIE-like gastrointestinal dysmotility and cachexia. J. Neurol. 256, 810-815

Hirano, M., Davidson, M., DiMauro, S. 2001. Mitochondria and the heart. Curr. Opin. Cardiol. 16, 201-210

Ingman M, Gyllensten U. 2003. Mitochondrial genome variation and evolutionary history of Australian and New Guinean aborigines. Genome Res. 13(7):16001606.

Led, D., et al., 2004. Clinical presentations of mitochondrial cardiomyopathies. Pediatr. Cardiol. 25, 443-450

Limongelli, G., et al., 2010. Prevalence and natural history of heart disease in adults 
with primary mitochondrial respiratory chain disease. Eur. J. Heart Fail. 12, 114121

Marín-García, J., et al., 2000. The complete sequence of mtDNA genes in idiopathic dilated cardiomyopathy shows novel missense and tRNA mutations. J. Card. Fail. 6, 321-329

Marín-García, J., et al., 2002. Severe mitochondrial cytopathy with complete A-V block, PEO, and mtDNA deletions. Pediatr. Neurol. 27, 213-216

Martinez, B., et al., 2001. Thyroid hormone regulates oxidative phosphorylation in the cerebral cortex and striatum of neonatal rats. J. Neurochem. 78,1054-1063

McFarland, R. et al., 2002. Multiple neonatal deaths due to a homoplasmic mitochondrial DNA mutation. Nat. Genet. 30, 145-146

McFarland, R., et al., 2004. Assigning pathogenicity to mitochondrial tRNA mutations: when 'definitely maybe' is not good enough. Trends Genet. 20, 591596

Moraes, C.T., et al., 1993. Atypical clinical presentations associated with the MELAS mutation at position 3243 of human mitochondrial DNA. Neuromuscul. Disord. $3,43-50$

Papadopoulou, L.C., et al., 1999. Fatal infantile cardioencephalomyopathy with COX deficiency and mutations in SCO2, a COX assembly gene. Nat. Genet. 23, 333337

Rorbach, J., et al. 2008. Overexpresion of human mitochondrial valyl tRNA synthetase can partially restore levels of cognate mt-tRNA ${ }^{\mathrm{Val}}$ carrying the pathogenic $\mathrm{C} 25 \mathrm{U}$ mutation. Nucleic. Acids. Res. 36, 3065-3074

Sacconi, S., et al., 2002. Complex neurologic syndrome associated with the G1606A mutation of mitochondrial DNA. Arch. Neurol. 59, 1013-1015 
Sciacco, M., Bonilla, E., 1996. Cytochemistry and immunocytochemistry of mitochondria in tissue sections. In: Attardi GM, Chomyn A, eds. Methods in enzymology. Vol. 264. Mitochondrial biogenesis and genetics. Part B. San Diego, CA: Academic Press, 509-521

Shimotake, T., Furukawa, T., Inoue, K., Iwai, I., Takeuchi, Y., 1998. Familial occurrence of intestinal obstruction in children with the syndrome of mitochondrial encephalomyopathy, lactic acidosis, and stroke-like episodes (MELAS). J. Pediatr. Surg. 33, 1837-1839

Sprinzl, M., et al., 1989. Compilation of tRNA sequences and sequences of tRNA genes. Nucleic. Acid. Res. R1-172 (suppl)

Tanji, K., et al., 2008. A novel tRNA ${ }^{\mathrm{Val}}$ mitochondrial DNA mutation causing MELAS. J. Neurol. Sci. 270, 23-27

Taylor, R.W., et al., 1996. MELAS associated with a mutation in the valine transfer RNA gene of mitochondrial DNA. Ann. Neurol. 40, 459-462

Taylor, R.W., et al., 2003. A homoplasmic mitochondrial transfer ribonucleic acid mutation as a cause of maternally inherited hypertrophic cardiomyopathy. J. Am. Coll. Cardiol. 41, 1786-1796

Tiranti, V., et al., 1998. A novel mutation in the mitochondrial tRNA ${ }^{\mathrm{Val}}$ gene associated with a complex neurological presentation. Ann. Neurol. 43, 98-101

Torroni, A., et al., 2003. Mitochondrial DNA haplogroups do not play a role in the variable phenotypic presentation of the A3243G mutation. Am. J. Hum. Genet. $72,1005-1012$

Tucker, E.J., Compton, A.G., Thorburn D.R., 2010. Recent advances in the genetics of mitochondrial encephalopathies. Curr. Neurol. Neurosci. Rep. 10, 277-285.

Wahbi, K., et al., 2010. Cardiac involvement is frequent in patients with the 
m.8344A $>$ G mutation of mitochondrial DNA. Neurology 74, 674-677

Yan, N., et al. 2010. A novel mitochondrial tRNA ${ }^{\mathrm{Val}} \mathrm{T} 1658 \mathrm{C}$ mutation identified in a CPEO family. Mol. Vis. 16, 1736-1742

Zeviani, M., et al., 1991. Maternally inherited myopathy and cardiomyopathy: association with mutation in mitochondrial DNA tRNA(Leu)(UUR). Lancet. $338,143-147$ 


\section{Figure captions}

Fig. 1. PCR-RFLP analyses of the mutations. PCR-amplified and radiolabeled mtDNA fragments were digested, separated by gel electrophoresis, exposed to a phosphorimager plate for $24 \mathrm{~h}$, and analyzed with image-quant software. Panel A: PCR-RFLP analysis of the m.1628C $>\mathrm{T}$ transition. Amplified fragments of 435 -bp are cut into 403-bp and 32-bp fragments after digestion with PvuII when harboring the wild-type molecule. Lanes: 1, uncut band control muscle; 2, cut band control muscle; 3 , uncut band patient 1 muscle; 4, cut band patient 1 muscle. Panel B: PCR-RFLP analysis of the m.1644G $>$ A mutation. Amplified fragments of 192-bp are cut into 133bp and 59-bp fragments after digestion with Tsp509I when harboring the m.1644G $>\mathrm{A}$ mutation. Lanes: 1, uncut band control muscle; 2, cut band control muscle; 3, uncut band patient 2 muscle; 4 , cut band patient 2 muscle.

\section{Fig. 2. Steady-state levels of tRNA ${ }^{\mathrm{Val}}$ in muscle from controls and patient 2.}

Total RNA extracted from skeletal muscle was analysed by Northern blotting using specific tRNA $^{\mathrm{Val}}$ and tRNA ${ }^{\text {Phe }}$ probes. Equal amounts of RNA $(5 \mu \mathrm{g})$ were fractionated on $12 \%$ polyacrylamide- $7 \mathrm{M}$ urea gels, transferred onto a nylon membrane, hybridized with the tRNA ${ }^{\mathrm{Val}}$ and $\mathrm{tRNA}{ }^{\text {Phe }}$ radiolabelled probes, and autoradiographed (left panels). Lane1, patient 1 RNA; Lane 2, control RNA 1; Lane 3, control RNA 2. Signals from two independent experiments for each sample were quantified by phosphoimager readings. The steady-state levels of $\mathrm{tRNA}^{\mathrm{Val}}$ normalized against $\mathrm{tRNA}{ }^{\text {Phe }}$ are shown on the right. Val/Phe-1, Val/Phe-2, and Val/Phe-3 correspond to the ratios of tRNA ${ }^{\mathrm{Val}}$ and tRNA $^{\text {Phe }}$ shown in the left hand panels in lanes 1,2 , and 3, respectively. 
Fig. 3. Schematic representation of the mt-tRNA ${ }^{\text {Val }}$ cloverleaf structure. Three sets of pathogenic mutations associated with MCMs are highlighted in red. The two mutations identified in the present study are highlighted in green. Other known mutations in the same mitochondrial $\mathrm{tRNA}^{\mathrm{Val}}$ but not related with $\mathrm{MCM}$ are also indicated. 